\title{
Wässrige Latices aus hochmolekularem Polyethylen durch katalytische Polymerisation**
}

Florian M. Bauers und Stefan Mecking*

Die Emulsionspolymerisation olefinischer Monomere zählt $\mathrm{zu}$ den bedeutendsten und auch vielseitigsten Polymerisationsverfahren. ${ }^{[1]}$ Als Produkte werden Polymerlatices erhalten, d.h. stabile wässrige Dispersionen von durch Tenside stabilisierten Polymer-Mikropartikeln mit Größen im Bereich von 50 bis $1000 \mathrm{~nm}$. Zahlreiche Anwendungen von Polymerlatices (z. B. in Beschichtungen aller Art) beruhen auf der Filmbildung beim Verdampfen des Dispersionsmediums. Die Umweltfreundlichkeit und die Unbrennbarkeit von Wasser sind dabei von besonderem Vorteil. Bislang werden Polymerlatices ausschließlich durch radikalische Polymerisation hergestellt.. ${ }^{[2]}$ Übergangsmetallkatalysierte Polymerisationen haben dagegen vergleichsweise wenig Aufmerksamkeit erhalten, da die für die kommerzielle Produktion von Polyolefinen verwendeten Katalysatoren auf der Basis früher Übergangsmetalle ${ }^{[3]}$ extrem empfindlich gegenüber Feuchtigkeit sind. Es wäre jedoch attraktiv, auch derartige Polymerisationen in Wasser durchzuführen, da zahlreiche Polymer-Mikrostrukturen nur durch katalytische Polymerisation zugänglich sind. Wir ${ }^{[4]}$ und andere ${ }^{[5]}$ berichteten kürzlich über die Polymerisation von Ethylen in Wasser durch neutrale Nickel(II)-Komplexe. ${ }^{[6,7]}$ Dabei wurden jedoch Dispersionen

[*] Dr. S. Mecking, Dipl.-Chem. F. M. Bauers

Institut für Makromolekulare Chemie und Freiburger Materialforschungszentrum der Albert-Ludwigs-Universität Freiburg Stefan-Meier-Straße 31, 79104 Freiburg (Deutschland) Fax: $(+49) 761-203-6319$ E-mail: mecking@uni-freiburg.de

[**] Diese Arbeit wurde von der BASF AG unterstützt. F.M.B. dankt der Deutschen Forschungsgemeinschaft für ein Stipendium (Graduiertenkolleg Strukturbildung in makromolekularen Systemen). Wir danken R. Thomann für die TEM-Aufnahmen und U. Westphal für die Durchführung der GPC-Untersuchungen. 
aus niedermolekularem Material mit einem Polymerisationsgrad von weniger als 100 erhalten $\left(M_{\mathrm{n}}\right.$ betrug typischerweise $\left.1000 \mathrm{~g} \mathrm{~mol}^{-1}\right)$. Um das besondere Eigenschaftsprofil von Latices nutzen zu können, ist die Synthese von höhermolekularen Polymeren (mit Kettenlängen, welche deutlich über der Entanglement-Grenze liegen) wünschenswert. Gleichzeitig müssen die komplexen Schritte der Partikelbildung während der Polymerisation zur Bildung stabiler Latices führen.

Unter Verwendung von Komplexen $\left[\left(\mathrm{X}^{\frown} \mathrm{O}\right) \mathrm{Ni}^{\mathrm{II}} \mathrm{R}(\mathrm{L})\right]$ mit $\mathrm{X}=\mathrm{P}($ Typ $\mathbf{1})$ und $\mathrm{X}=\mathrm{N}($ Typ $2, \mathrm{~N} \frown \mathrm{O}=$ Salicylaldimin) sind in herkömmlichen Polymerisationen in unpolaren organischen Reaktionsmedien Materialien mit einer ähnlichen maximalen gewichtsmittleren Molmasse $\left(M_{\mathrm{w}}\right)$ zugänglich. ${ }^{[8,9]}$ Im Unterschied dazu können durch wässrige Polymerisationen mit 2 Polyethylene erhalten werden, deren $M_{\mathrm{w}}$ - und $M_{\mathrm{n}}$ Werte wesentlich höher sind als die der bislang mit Komplexen $\mathbf{1}$ in Wasser erhaltenen niedermolekularen Materialien. ${ }^{[4 b]}$ Hinsichtlich des Partikelbildungsprozesses ist ein Vergleich mit der intensiv untersuchten radikalischen Emulsionspolymerisation hilfreich. ${ }^{10]}$ In der klassischen Emulsionspolymerisation werden wasserlösliche Initiatoren verwendet. Die ersten Schritte des Kettenwachstums führen zu zunächst wasserlöslichen Oligoradikalen. Die Nucleierung von Partikeln kann durch ein „Kollabieren“ der wachsenden Kette auf sich selber nach Erreichen einer gewissen Länge oder durch vorherigen Eintritt des Oligoradikals in eine Tensidmicelle erfolgen. Ähnliche Mechanismen scheinen im Falle der bereits erwähnten Ethylenpolymerisation in Emulsion durch hydrophil modifizierte, wasserlösliche Komplexe des Typs 1, welche zu stabilen Latices aus niedermolekularem Material führt, vorzuliegen. ${ }^{[4]}$ Eine gewisse Wasserempfindlichkeit ${ }^{[4 \mathrm{~b}, 9 \mathrm{c}]}$ der Salicylaldiminkomplexe (Typ 2) könnte für einen analogen Ansatz jedoch von Nachteil sein.

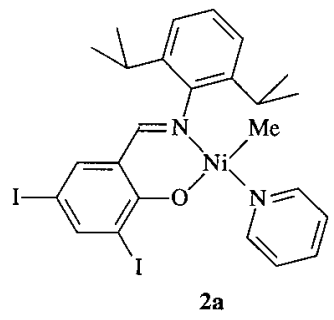

Als ein alternatives Konzept zur Bildung einer großen Zahl von Latexpartikeln wähend der Polymerisation wurde die feine Verteilung der Katalysatorvorstufe in Form einer Lösung eines lipophilen Komplexes (2a) in Submikron-großen Tröpfchen eines Kohlenwasserstoffes, welche in der kontinuierlichen wässrigen Phase dispergiert sind, verfolgt. ${ }^{[14]}$ Eine Mischung aus Wasser, Tensid und einer Lösung des Komplexes in einer kleinen Menge Kohlenwasserstoff (Toluol und eine geringe Menge Hexadecan als hydrophobes Additiv $\left.{ }^{[11]}\right)$ wurde hohen Scherkräften ausgesetzt, welche mittels Ultraschall oder durch einen modifizierten Hochdruckhomogenisator erzeugt wurden. Dadurch wurden Miniemulsionen erhalten, welche aus einer großen Zahl von die Katalysatorvorstufe enthaltenden hydrophoben Tröpfchen bestehen (Durchmesser ca. $100 \mathrm{~nm}, 10^{16}-10^{17}$ Tröpfchen $\mathrm{L}^{-1}$ ). Die Umsetzung mit Ethylen in einem Druckreaktor führt zur Bildung stabiler Polyethylenlatices (Tabelle 1). Die gelpermeationschromatographische (GPC-) Analyse der aus diesen Dispersionen isolierten Polymere ergab, dass es sich ausschließlich um hochmolekulares Polyethylen mit Molmassen bis zu $M_{\mathrm{w}}=4 \times 10^{5} \mathrm{~g} \mathrm{~mol}^{-1}$ bei relativ engen Molmassenverteilungen handelt. Die durch Lichtstreuung bestimmten mittleren Partikelgrößen der Latices liegen im Bereich von 90 bis 350 nm. ${ }^{[12]}$ Die hier beschriebene Ethylenpolymerisation unterscheidet sich von einer typischen (radikalischen) Polymerisation einer Miniemulsion eines flüssigen Monomers. Im vorliegenden Fall wird gasförmiges Ethylen kontinuierlich zum Reaktionsgemisch zudosiert, ausgehend von einer Miniemulsion der Katalysatorlösung. Das typische Merkmal einer „Miniemulsionspolymerisation“ im engeren Sinne, die Polymerisation von Miniemulsionströpfchen des Monomers zu Polymerpartikeln derselben Größe, liegt hier also nicht vor.

Die erhaltenen Polymerlatices sind über Wochen oder länger stabil. Als Tensid sind verschiedene anionische oder nichtionische Verbindungen geeignet, die im verwendeten Miniemulsionssystem mit dem Katalysator verträglich sind und die Bildung stabiler Latices ermöglichen (Tabelle 1). Wie erwartet führt eine Verringerung der Tensidmenge unter ansonsten gleichen Bedingungen zu einer Erhöhung der mittleren Partikelgröße (Nr. 2-4). Die bei Verwendung des Hochdruckhomogenisators auftretenden niedrigeren Aktivitäten (Nr. 7-9) im Vergleich zur denen, die bei Scherung mittels Ultraschall erhalten werden, sind wahrscheinlich auf eine Desaktivierung der Katalysatorvorstufe zurückzuführen, da mit der verwendeten Versuchsanordnung eine längere Zeit für die Miniemulgierung erforderlich war.

Tabelle 1. Polymerisationsbedingungen ${ }^{[a]}$ und -ergebnisse.

\begin{tabular}{|c|c|c|c|c|c|c|c|c|c|c|c|}
\hline Nr. & $\begin{array}{l}n \text { (Kat.) } \\
{[\mu \mathrm{mol}]}\end{array}$ & $\begin{array}{l}\text { Toluol } \\
\text { [Vol.-\%] }\end{array}$ & $\begin{array}{l}\mathrm{C}_{16} \mathrm{H}_{34} \\
\text { [Vol.-\%] }\end{array}$ & $\operatorname{Typ}^{[b]}$ & $\begin{array}{l}\text { Tensid } \\
\qquad c\left[\mathrm{mmol} \mathrm{L}^{-1}\right]\end{array}$ & $\begin{array}{l}\text { Emulgier- } \\
\text { methode }^{[c]}\end{array}$ & $\mathrm{TON}^{[\mathrm{d}]}$ & $\begin{array}{l}M_{\mathrm{n}} \\
{\left[\mathrm{g} \mathrm{mol}^{-1}\right]}\end{array}$ & $\begin{array}{l}M_{\mathrm{w}} \\
{\left[\mathrm{g} \mathrm{mol}^{-1}\right]}\end{array}$ & $M_{\mathrm{w}} / M_{\mathrm{n}}$ & $\begin{array}{l}\bar{d}^{[\mathrm{e}]} \\
{[\mathrm{nm}]}\end{array}$ \\
\hline 1 & 25 & 1 & 2 & SDS & 17 & US & 2515 & $1.4 \times 10^{5}$ & $3.2 \times 10^{5}$ & 2.3 & 330 \\
\hline 2 & 23 & 1 & 0.3 & SDS & 35 & US & 1206 & $1.2 \times 10^{5}$ & $3.3 \times 10^{5}$ & 2.7 & 220 \\
\hline 3 & 36 & 1 & 0.3 & SDS & 17 & US & 1135 & $8.5 \times 10^{4}$ & $2.6 \times 10^{5}$ & 3.1 & 260 \\
\hline 4 & 29 & 1 & 0.3 & SDS & 5 & US & 1230 & $1.0 \times 10^{5}$ & $4.5 \times 10^{5}$ & 4.5 & $(485)^{[\mathrm{f}]}$ \\
\hline 5 & 36 & 1 & 0.3 & Trit & 11 & US & 959 & $8.9 \times 10^{4}$ & $1.9 \times 10^{5}$ & 2.1 & 100 \\
\hline 6 & 32 & 1 & 0.3 & K30 & 18 & US & 990 & $9.0 \times 10^{4}$ & $2.5 \times 10^{5}$ & 2.8 & 240 \\
\hline 8 & 32 & 1 & 0.3 & SDS & 17 & HP & 75 & $2.0 \times 10^{4}$ & $3.4 \times 10^{4}$ & 1.7 & 90 \\
\hline 9 & 44 & 4 & 1 & Lut & 5 & HP & 265 & $1.6 \times 10^{4}$ & $2.4 \times 10^{4}$ & 1.5 & 250 \\
\hline
\end{tabular}

[a] $30^{\circ} \mathrm{C}, 45$ bar konstanter Ethylendruck, $2 \mathrm{~h}$ Reaktionszeit. Gesamtvolumen Wasser und organische Lösungsmittel: $100 \mathrm{~mL}$. [b] SDS = Natriumdodecylsulfat, Lut = Lutensol AT $80\left(\mathrm{C}_{16}-\mathrm{C}_{18}\right.$-Fettalkohol-Ethoxylat mit durchschittlich 80 EO-Einheiten; BASF), Trit = Triton X-100 (Isooctylphenololigoethoxylate), K30 = Emulgator K30/40 (Bayer; Natriumalkylsulfonate). [c] US = Ultraschall, HP= Hochdruckhomogenisator (siehe Experimentelles). [d] In Mol Ethylen pro Mol Ni. [e] Mittlerer Durchmesser der Latex-Partikel, bestimmt durch Lichtstreuungsmessungen. [f] Instabiler Latex, teilweise Koagulation. 
Die Latexpartikel wurden mittels Transmissionselektronenmikroskopie (TEM; Abbildung 1) visualisiert. Im Vergleich zu typischen glatten, runden Partikeln von amorphem

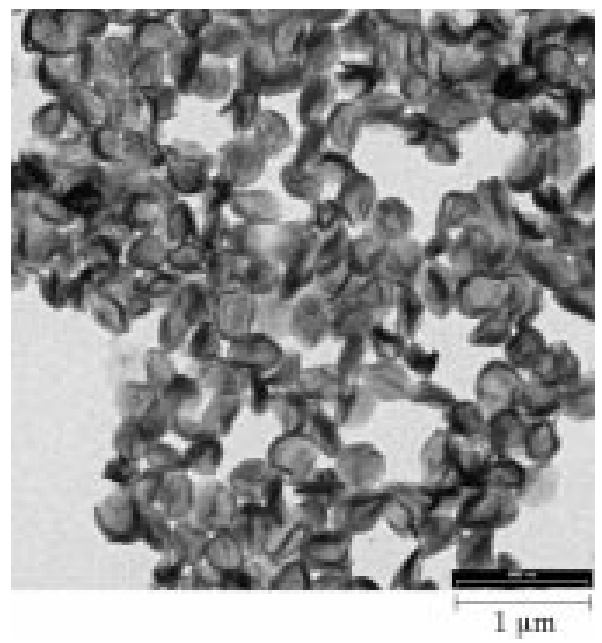

Abbildung 1. TEM-Aufnahme von Latexpartikeln.

Polystyrol als einem durch radikalische Emulsionspolymerisation erhaltenen Kohlenwasserstoff-Polymer können die rauhe Oberfläche und die nichtsphärische Gestalt der Polyethylenpartikel auf dessen hohe Kristallinität zurückgeführt werden $\left(39-51 \%\right.$ laut Differenzkalorimetrie (DSC); $T_{\mathrm{m}}=$ $\left.120-130{ }^{\circ} \mathrm{C}\right){ }^{[13]}$

\section{Experimentelles}

Allgemeines: Der Komplex 2a wurde wie bereits beschrieben synthetisiert. ${ }^{[4 b]}$ Die Verbindung wurde mittels Schlenk-Technik unter Argon gehandhabt. Deionisiertes Wasser wurde vor der Verwendung entgast. Die Hochtemperatur-GPC-Analyse wurde in 1,2,4-Trichlorbenzol bei $140^{\circ} \mathrm{C}$ auf einem PL-220-Gerät mit Mixed-Bed-PL-Säulen durchgeführt. Die angebenen Daten sind auf lineares Polyethylen bezogen (universelle Kalibrierung). Die Untersuchungen zur dynamischen Lichtstreuung wurden an verdünnten Dispersionen mit einem Malvern Particle Sizer durchgeführt. Die TEM-Bilder wurden an einem LEO-912-Omega-Mikroskop bei $120 \mathrm{kV}$ Beschleunigungsspannung aufgenommen. Die DSCDaten wurden im zweiten Aufheizcyclus bei $10 \mathrm{~K} \mathrm{~min}^{-1}$ ermittelt.

Polymerisation: Die Katalysatorvorstufe wurde in den angegebenen Mengen (Tabelle 1) Toluol und Hexadecan gelöst. Das Tensid und etwa $100 \mathrm{~mL}$ Wasser wurden unter Rühren zugegeben. Die Homogenisierung erfolgte unter Argon mit einem direkt mit dem Polymerisationsreaktor verbundenen Hochdruckhomogenisator (modifzierte Version eines EmulsiFlex-C5-Gerätes von Avestin Inc.; 500-1000 bar) oder alternativ durch Ultraschallbehandlung (Bandelin HD2200 mit KE76-Spitze; 2 min bei $120 \mathrm{~W})$. Die erhaltenen Miniemulsionen wurden in einen mechanisch gerührten 250-mL-Druckreaktor mit Doppelmantel überführt, und ein konstanter Ethylendruck von 45 bar wurde aufgepresst. Die Reaktionstemperatur von $30^{\circ} \mathrm{C}$ wurde über einen in das Reaktionsgemisch eintauchenden Thermofühler verfolgt und automatisch reguliert. Nach der vorgegebenen Reaktionszeit wurde das überschüssige Ethylen abgelassen. Der erhaltene Latex wurde durch Glaswolle filtriert. Zur Bestimmung der Ausbeute und zur weiteren Analyse wurde ein abgemessener Anteil des Latex aus Methanol gefällt.

[1] a) Emulsion Polymerization and Emulsion Polymers (Hrsg.: P. A. Lovell, M. S. El-Aasser), Wiley, Chichester, 1997; b) Wässrige Polymerdispersionen (Hrsg.: D. Distler), VCH, Weinheim, 1999; c) G.
Lagaly, O. Schulz, R. Zimehl, Dispersionen und Emulsionen, Steinkopff, Darmstadt, 1997.

[2] Im Labormaßstab wurden Latices auch nach anderen Reaktionstypen hergestellt: a) D. R. Weyenberg, D. E. Findlay, J. Cekada, A. E. Bey, J. Polym. Sci. C 1969, 27, 27-34 (ionische ringöffnende Polymerisation cyclischer Siloxane); b) K. Landfester, F. Tiarks, H.-P. Hentze, M. Antonietti, Macromol. Chem. Phys. 2000, 201, 1-5 (Polyaddition).

[3] a) Ziegler Catalysts (Hrsg.: G. Fink, R. Mülhaupt, H. H. Brintzinger), Springer, Berlin, 1995; b) H. H. Brintzinger, D. Fischer, R. Mülhaupt, B. Rieger, R. Waymouth, Angew. Chem. 1995, 107, 1255-83; Angew. Chem. Int. Ed. Engl. 1995, 34, 1143-1170; c) G. J. P. Britovsek, V. C. Gibson, D. F. Wass, Angew. Chem. 1999, 111, 448-468; Angew. Chem. Int. Ed. 1999, 38, 428-447; d) W. Kaminsky, M. Arndt, Adv. Polym. Sci. 1997, 127, 143-187.

[4] a) A. Held, F. M. Bauers, S. Mecking, Chem. Commun. 2000, 301 302; b) F. M. Bauers, S. Mecking, Macromolecules 2001, 34, 1165 1171.

[5] Kurz nach unserem ersten Bericht ${ }^{[4 a]}$ wurden die Ergebnisse von unabhängigen Untersuchungen zur $\mathrm{Ni}^{\mathrm{II}}$-katalysierten Polymerisation in Wasser als Reaktionsmedium publiziert: A. Tomov, J.-P. Broyer, R. Spitz, Macromol. Symp. 2000, 150, 53-58. Siehe auch: A. Tomov, R. Spitz, T. Saudemont, X. Drujon (Elf Atochem), WO 00/20464, 2000 [Chem. Abstr. 2000, 132, 279651a].

[6] Polymerisation von Ethylen zu hochmolekularem amorphem Polyethylen in Suspension: a) A. Held, S. Mecking, Chem. Eur. J. 2000, 6, $4623-4629$, zit. Lit. Sehr langsame ((1 mol Ethylen) $(1 \mathrm{~mol} \mathrm{Rh})^{-1}$ pro Tag) Rh-katalysierte Ethylenpolymerisation in wässriger Suspension: b) L. Wang, R. S. Lu, R. Bau, T. C. Flood, J. Am. Chem. Soc. 1993, 115, 6999-7000. Wässrige katalytische Polymerisationen anderer olefinischer Monomere als Ethylen: c) R. E. Rinehart, H. P. Smith, H. S. Witt, H. Romeyn, J. Am. Chem. Soc. 1961, 83, 4864-4865; d) G. Natta, G. Dall'Asta, L. Porri, Makromol. Chem. 1965, 81, 253-257; e) R. E. Rinehart, J. Polym. Sci. C 1969, 27, 7-25; f) B. M. Novak, R. H. Grubbs, J. Am. Chem. Soc. 1988, 110, 7542-7543; g) D. M. Lynn, B. Mohr, R. H. Grubbs, J. Am. Chem. Soc. 1998, 120, 1627-1628; h) W. Reppe, A. Magin, US-A 2577208, 1951 [Chem. Abstr. 1952, 46, 6143b]; i) Z. Jiang, A. Sen, Macromolecules 1994, 27, 7215-7216; j) G. Verspui, G. Papadogianakis, R. A. Sheldon, Chem. Commun. 1998, 401-402; k) E. Lindner, M. Schmid, J. Wald, J. A. Queisser, M. Geprägs, P. Wegner, C. Nachtigal, J. Organomet. Chem. 2000, 602, $173-187$.

[7] Übersichtsartikel zur Olefinpolymerisation durch Komplexe später Übergangsmetalle: a) S. Mecking, Coord. Chem. Rev. 2000, 203, 325 351; b) S. D. Ittel, L. K. Johnson, M. Brookhart, Chem. Rev. 2000, 100, 1169-1204; c) S. Mecking, Angew. Chem. 2001, 113, 550-557; Angew. Chem. Int. Ed. 2001, 40, 534-540; d) Lit. [3c].

[8] a) K. A. Ostoja-Starzewski, J. Witte, Angew. Chem. 1987, 99, 76-77; Angew. Chem. Int. Ed. Engl. 1987, 26, 63-64; b) U. Klabunde, S. D. Ittel, J. Mol. Catal. 1987, 41, 123-134.

[9] a) L. K. Johnson, A. M. A. Bennett, S. D. Ittel, L. Wang, A. Parthasarathy, E. Hauptman, R. D. Simpson, J. Feldman, E. B. Coughlin (DuPont), WO 98/30609, 1998 [Chem. Abstr. 1998, 129, 149362j]; b) C. Wang, S. Friedrich, T. R. Younkin, R. T. Li, R. H. Grubbs, D. A. Bansleben, M. W. Day, Organometallics 1998, 17, 3149-3151; c) T. R. Younkin, E. F. Connor, J. I. Henderson, S. K. Friedrich, R. H. Grubbs, D. A. Bansleben, Science 2000, 287, 460-462.

[10] Die unterschiedlichen Mechanismen von Kettenwachstum, -start und -übertragung in radikalischen und katalytischen Polymerisationen können die komplexen Vorgänge der Partikelnucleierung und des Partikelwachstums beeinflussen. Der hier gezogene allgemeine Vergleich ist jedoch in diesem Rahmen sinnvoll.

[11] $\mathrm{Zu}$ Miniemulsionen und der Rolle des hydrophoben Additivs siehe: a) E. D. Sudol, M. S. El-Aasser in Emulsion Polymerization and Emulsion Polymers (Hrsg.: P. A. Lovell, M. S. El-Aasser), Wiley, Chichester, 1997, S. 699-722; b) K. Landfester, N. Bechthold, F. Tiarks, M. Antonietti, Macromolecules 1999, 32, 5222-5228, zit. Lit.

[12] Bezüglich der Entwicklung der Partikelzahlen während der Polymerisation wird die Interpretation der Ergebnisse aufgrund der Notwendigkeit, die trüben Proben für die Lichtstreuung stark zu verdünnen, erschwert. Im Falle der Katalysator-Miniemulsionen kann 
dies zu Abweichungen vom Ausgangszustand der (Flüssig/flüssig-) Emulsion führen.

[13] Die Polyethylene sind wie auch die in herkömmlichen Polymerisationen in organischen Medien mit Katalysatoren vom Typ 2 erhaltenen Materialien ${ }^{[9]}$ mäßig verzweigt. In dem aus wässrigen Dispersionen isolierten Polymer wurden ${ }^{1} \mathrm{H}$ - und ${ }^{13} \mathrm{C}$-NMR-spektroskopisch etwa 20 Verzweigungen auf 1000 Kohlenstoffatome nachgewiesen (überwiegend Methyl-Verzweigungen). Im Vergleich zu linearem Polyethylen ist die Kristallinität leicht verringert.

[14] Anmerkung bei der Überarbeitung des Manuskriptes (23. Mai 2001): Nach der Einreichung dieser Arbeit wurde über die Herstellung von Latices aus niedermolekularem Material durch Ethylenpolymerisation unter Einsatz von Miniemulsionen wasserstabiler Nickel(II)Komplexe berichtet: R. Soula, C. Novat, A. Tomov, R. Spitz, J. Claverie, X. Drujon, J. Malinge, T. Saudemont, Macromolecules 2001, $34,2022-2026$. 